\title{
Variaciones espacio-temporales del ensamble de peces de un sistema fluvial de bajo orden del centro-sur de Chile
}

\author{
Spatial and temporal variations of the fish assemblage of a low order fluvial system \\ from central-south of Chile
}

EVELYN HABIT ${ }^{1}$, PEDRO VICTORIANO² \& AMADORA RODRÍGUEZ-RUIZ ${ }^{3}$

\author{
${ }^{1}$ Departamento de Química, Facultad de Ciencias, Universidad del Bío-Bío, Casilla 5-C, Concepción, y \\ Centro de Ciencias Ambientales EULA-Chile, Universidad de Concepción, Chile; \\ e-mail: ehabit@ubiobio.cl \\ ${ }^{2}$ Departamento de Zoología, Facultad de Ciencias Naturales y Oceanográficas, \\ Universidad de Concepción, Chile \\ ${ }^{3}$ Departamento de Biología Vegetal y Ecología, Facultad de Biología, \\ Universidad de Sevilla, España
}

\begin{abstract}
RESUMEN
El estero Nonguén (orden 3), tributario de la zona inferior del río Andalién (región del Bío-Bío), presenta una marcada diferencia entre su parte alta ritral, bien conservada y con un bosque secundario de Nothofagus, y su parte baja potamal, de uso urbano y de aguas con contaminación orgánica. Este contraste podría implicar la alteración de los patrones normales detectados en la ictiofauna chilena descritos para diversos sistemas fluviales. Tales patrones consisten en un aumento del número de especies, individuos y diversidad en el sentido de la corriente, así como en un incremento de las tallas corporales de los individuos que habitan las zonas bajas. Se estudió la composición específica de peces del estero Nonguén y sus patrones comunitarios a lo largo del sistema fluvial en un ciclo anual. Se encontró un incremento en la riqueza específica, desde cuatro especies en el ritrón a diez en el sector de desembocadura. Tanto la diversidad, abundancia y distribución de los peces varió según la época del año, reflejando los desplazamientos asociados a la ontogenia, reproducción y alimentación de la fauna íctica. Las estaciones de la zona media y baja del estero presentaron un ensamble con individuos de menor tamaño que los que se encuentran en la cabecera, consistentemente con la dominancia de salmónidos de mayor talla en la parte alta. Los resultados sugieren que: (i) la alteración del área potamal no modifica significativamente los patrones característicos de la ictiofauna de sistemas fluviales, y que (ii) el estero Nonguén es un sistema de reproducción y crianza de juveniles de especies nativas e introducidas, las cuales conforman asociaciones permanentes en este sistema fluvial.
\end{abstract}

Palabras clave: ictiofauna, diversidad, variaciones espacio-temporales, ensamble, sistema fluvial.

\begin{abstract}
The Nonguén stream (order 3) is a lower zone tributary of the Andalién river coastal mountain basin (Bio-Bío region) which displays a marked difference between its rithral sector (well-conserved and with a secondary Nothofagus forest) and its potamal area (urbanly-used and organically polluted waters). This contrast could imply an alteration in the normal pattern of Chilean ichthyofauna, as described for a variety of fluvial systems in the country: a downstream increase in numbers of species, individuals, and diversity downstream, as well as increased corporal sizes of individuals in the lower zones of the river. Species composition of fishes of the Nonguén stream and their community patterns along the fluvial system within an annual cycle have been studied. The results show a high number of species in the lower parts, i.e. four species in the rithron and ten in the river mouth. The diversity, abundance and distribution of the fishes varied according to the season of the year, reflecting displacements associated with the fauna's ontogeny, reproduction and feeding. The sampling stations of the middle and lower zones of the river presented an assemblage with smaller individuals than those found at the head of the river, with the consistent dominance of larger salmonidae in the upper zone. Accordingly, it is suggested that: (i) the alteration of the potamal area does not significantly modify the characteristic patterns of ichthyofauna in fluvial systems, and (ii) the Nonguén stream is a reproduction and growth system for juveniles of both native and introduced species which make up permanent associations in the fluvial system.
\end{abstract}

Key words: ichthyofauna, diversity, streams, spatial-temporal variations, assemblage, fluvial system. 


\section{INTRODUCCIÓN}

La ictiofauna de los sistemas fluviales se distribuye heterogéneamente a lo largo de su desarrollo, presentando patrones comunitarios tales como el incremento de la riqueza específica, abundancia y diversidad en el sentido de la corriente (Illies \& Botosaneanu 1963, Vannote et al. 1980, Welcomme 1985). Estos patrones han sido explicados por la mayor disponibilidad de hábitats y nivel de trofía de las aguas en las zonas bajas de los ríos (Welcomme 1985). A esto se asocia un aumento de las tallas corporales de los ensambles de los sectores potamales debido a un incremento de profundidad aguas abajo, mientras los sectores ritrales se mantienen como áreas de reproducción y crianza (Campos 1985, Callow \& Petts 1994).

Por otra parte, la ictiofauna de los sistemas fluviales tiende a presentar cambios en sus patrones espaciales de distribución y uso del hábitat, debido a variaciones temporales asociadas a la reproducción y/o búsqueda de alimento (Wootton 1990, Callow \& Petts 1994). La determinación de estos patrones espaciales a mesoescala y su variación temporal, es un aspecto fundamental para la gestión de la conservación biológica, puesto que si bien, en muchos casos las especies ictiofaunísticas ocupan la totalidad del río, ocurre una repartición espacio-temporal compleja de sus procesos biológicos (i.e., reproducción y crian$\mathrm{za})$.

En Chile, los ríos andinos (cuencas originadas en la Cordillera de Los Andes), y particularmente los ríos de origen costero (cuencas de la Cordillera de La Costa), presentan un perfil longitudinal marcadamente cóncavo (Campos et al. 1993, Jaque 1995), lo que genera la existencia de hábitats ritrales y potamales en cortas distancias (Campos 1985). En los sistemas costeros se ha desarrollado una ictiofauna similar a la de los ríos andinos, la cual presenta características particulares que la distingue de los peces del resto de Sudamérica (Campos et al. 1993), tales como una baja riqueza de especies y abundancia poblacional, pequeño tamaño corporal y alto grado de endemismo (Campos 1970, Arratia 1978, Campos et al. 1993, Vila et al. 1999a, Dyer 2000).

Las cuencas costeras de la Octava Región de Chile constituyen unidades hidrográficas de gran valor biogeográfico y de conservación biológica, puesto que albergan una alta proporción de la diversidad nativa total en áreas relativamente restringidas (Ruiz 1993). Sin embargo, el grado de intervención antrópica (Habit \& Parra 2001) ha generado un proceso de disminución progresiva en la distribución y abundancia de su ictiofauna (Arratia 1983, Vila et al. 1999a), lo cual puede alterar sus patrones naturales de zonación ictiofaunística. Una de estas cuencas costeras es la del río Andalién ubicada en la vertiente occidental de la Cordillera de La Costa, entre los $36^{\circ} 42^{\prime}$ y $\operatorname{los} 36^{\circ} 56^{\prime}$ S. En particular, el curso inferior del estero Nonguén $\left(36^{\circ} 49^{\prime}-36^{\circ} 54^{\prime} \mathrm{S}\right.$, $72^{\circ} 57^{\prime}-73^{\circ} 01^{\prime}$ O), último tributario del Andalién, atraviesa directamente por un sector de alta densidad urbana en la ciudad de Concepción (Fig. 1), lo que ha implicado fuertes alteraciones en la morfología y calidad del agua de su zona baja por cambios en el uso del suelo e ingreso de contaminantes al curso fluvial.

El estero Nonguén es un sistema fluvial de orden 3 (EULA 2002), $15 \mathrm{~km}$ de longitud y $44 \mathrm{~km}^{2}$ de superficie de cuenca hidrográfica (Jaque 1995).

Este sistema es usado para generación de agua potable en su zona alta y recepción de efluentes domésticos en la zona inferior. Esto genera un ingreso de materia orgánica al estero por el aporte de residuos líquidos y sólidos. La cuenca del estero Nonguén presenta otros problemas ambientales que incluyen deforestación y urbanización en suelos no aptos de su zona media e inferior (Jaque 1994). Sin embargo, a diferencia de la mayoría de las cuencas costeras de la zona centrosur de Chile, ésta mantiene en su cabecera un bosque secundario de Nothofagus que por su extensión, naturalidad y grado de aislamiento, adquiere un alto valor ecológico y ambiental.

Dado el contraste de los hábitats presentes entre la zona alta y baja del río, se planteó como hipótesis que el aumento característico en el número de especies, individuos, diversidad y talla corporal desde la zona ritral a la potamal (Callow $\&$ Petts 1994), es alterado por la intervención a la que se encuentra sometida la cuenca en sus tramos medio e inferior. Los objetivos de este trabajo son evaluar las variaciones espacio-temporales de la composición, riqueza específica, abundancia, biomasa y tallas corporales del ensamble de peces del estero Nonguén (ritrón a desembocadura) en un ciclo anual.

\section{MATERIALES Y MÉTODOS}

Se estudió la composición específica de peces del estero Nonguén y sus patrones comunitarios a lo largo del sistema fluvial en un ciclo anual, y se determinaron los tamaños corporales del ensamble. Se realizaron cuatro muestreos a lo largo del estero en marzo de 1999 (otoño), julio de 1999 (invierno), noviembre de 1999 (primavera) y febrero de 2000 (verano). En cada uno de ellos se muestrearon cinco estaciones ubicadas desde el nacimiento del estero hasta su desembocadura 
(Fig. 1). La zona alta del Nonguén (altitud máxima $430 \mathrm{~m}$; EULA 2002) constituye un típico ritrón sensu Welcomme (1985) (estación E-1), es decir un sector de gran velocidad de corriente (> $0,5 \mathrm{~m} \mathrm{~s}^{-1}$ ), substrato rocoso de bolones y aguas oxigenadas de baja temperatura (Tabla 1). La parte baja (estaciones E-4 y E-5) constituye un ambiente de potamón, es decir un área de baja velocidad de corriente $\left(<0,5 \mathrm{~m} \mathrm{~s}^{-1}\right)$, substrato de arenas, mayor conductividad y presencia de macrófitas. El tramo medio del estero (estaciones E-2 y E-3) corresponde a una zona de transición, en que el ambiente se estructura en parches consecutivos de áreas con características ritrales y otras más bien potamales.

El entorno varía a lo largo del río desde riberas con vegetación nativa en la estación E-1, a áreas de cultivos y plantaciones (estación E-2), y ambientes netamente urbanos desde la estación E-3 hasta la desembocadura (estación E-5). La cabecera del río mantiene un relicto del bosque caducifolio de Concepción, caracterizado por especies de Nothofagus (particularmente $N$. dombeyi y $N$. obliqua) y elementos representativos de la flora regional como Criptocarya alba, Aetoxicon punctatum, Peumus boldus, Persea lingue, Podocarpus saligna y Laurelia sempervirens (EULA 2002). En la zona baja el cauce es alterado anualmente (semi-canalizado) para evitar inundaciones en las poblaciones aledañas.

Dado que los principales usos del sistema fluvial en la parte baja del estero provocan un ingreso de materia orgánica (residuos líquidos y sólidos domésticos), se utilizaron como indicadores de calidad del agua la temperatura $\left(0,1{ }^{\circ} \mathrm{C}\right.$ de precisión), pH (pHmetro portátil Cole Parmer), conductividad (conductivímetro Hanna) y oxígeno disuelto (oxigenómetro Hanna). Se incluyen además, a modo de referencia, mediciones realizadas posteriormente (marzo del 2002) de nutrientes, $\mathrm{DBO}_{5}$ y colimetría (EULA 2002). Se registraron también variables estructurales del hábitat, tales como profundidad media y máxima, ancho del cauce, tipo de sustrato, presencia ausencia de vegetación acuática y tipo de entorno al río (García de Jalón et al. 1993).

Debido a la pequeña envergadura del sistema fluvial se utilizó como único arte de pesca para la caracterización de la ictiofauna un equipo EFKO de pesca eléctrica. Para estandarizar las capturas, se recorrieron $50 \mathrm{~m}$ de largo en cada estación de muestreo, barriendo todo el ancho del cauce. La unidad de esfuerzo considerada fue de $50 \mathrm{~m}$ de longitud del estero y $20 \mathrm{~min}$ de pesca. Los peces capturados fueron mantenidos vivos en recipientes aireados y fueron identificados in situ hasta

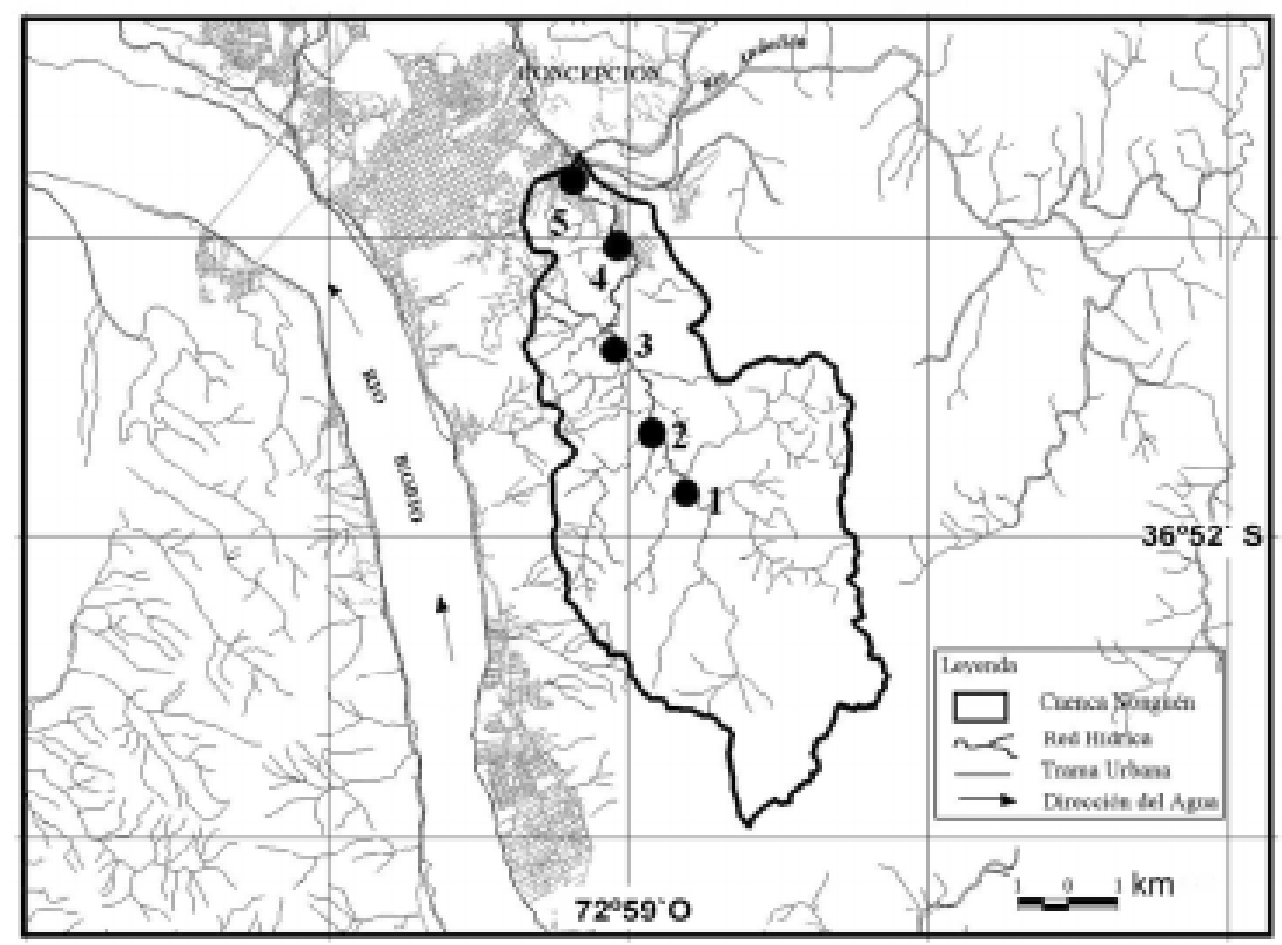

Fig. 1: Ubicación geográfica de la cuenca del estero Nonguén y localización de las estaciones de muestreo.

Geographic position of the Nonguén stream basin and position of the sampling stations. 
nivel de especie. A cada ejemplar se le midió la longitud total (vernier $0,1 \mathrm{~mm}$ de precisión) y el peso total (balanza digital Ohaus; $0,01 \mathrm{~g}$ de precisión). Posteriormente, todos los ejemplares fueron devueltos a su hábitat.

Se estudió la composición específica y la diversidad del ensamble de peces respecto al eje longitudinal del estero y al ciclo anual. Los cambios estacionales en el rango de abundancias de las distintas especies por estación de muestreo se analizaron mediante la prueba de KolmogorovSmirnov (Z). La diversidad fue evaluada a través del índice de Shannon-WeaverWiener: $\mathrm{H}=\Sigma \mathrm{p}_{\mathrm{i}}$ * $\log _{2} \mathrm{p}_{\mathrm{i}}$, donde $\mathrm{p}_{\mathrm{i}}=\mathrm{n}_{\mathrm{i}} / \mathrm{N}$, donde $\mathrm{n}_{\mathrm{i}}$ es el número de individuos por especie y $\mathrm{N}$ el número total de individuos. Para detectar posibles diferencias en la diversidad íctica a lo largo del río se aplicó la prueba no paramétrica U de Mann-Whitney.

Con el fin de estudiar las posibles diferencias, tanto espaciales (cabecera-desembocadura) como temporales (épocas del año), en cuanto al rango de talla del ensamble, se aplicó un análisis de varianza (ANOVA) de dos vías, mediante el paquete estadístico SPSS (SPSS Inc. 1998), siendo los factores fijos (Bennington \& Thayne 1994) la época del año, estación de muestreo y época del año x estación de muestreo. Las diferencias significativas entre cada par de factores consecutivos se contrastaron posteriormente con las comparaciones de Bonferroni. La longitud total fue transformada mediante $\log _{10}$, comprobándose la normalidad y homocedasticidad de las varianzas para cada esta- ción de muestreo, época del año y especie mediante el contraste de Levene. En todos los análisis se aceptó un nivel de significancia de 0,05. Finalmente, se determinaron también las variaciones en la abundancia (cpue) y biomasa (bpue) de peces del estero expresadas por unidad de esfuerzo, las que fueron relacionadas con las diferentes variables estructurales del hábitat, utilizando un análisis de regresión múltiple (Zar 1984).

\section{RESULTADOS}

La calidad del agua manifiesta una tendencia al incremento de la temperatura y conductividad aguas abajo, al mismo tiempo que existen valores menores de oxígeno disuelto. La mayor variación se observó en la colimetría y nutrientes (fósforo y nitrógeno), los cuales aumentan notoriamente hacia la desembocadura (Tabla 1).

Se capturó un total de 12 especies de peces, nueve nativas y tres introducidas (Tabla 2). De acuerdo con las categorías de conservación a nivel regional (Campos et al. 1998), tres especies se encuentran en "peligro de extinción" (Percilia irwini Eigenmann, 1927, Bullokia maldonadoi (Eigenmann, 1927) y Nematogenys inermis (Guichenot, 1848), en tanto que las restantes corresponden a especies "vulnerables".

Las capturas por unidad de esfuerzo (cpue) mostraron diferencias entre las distintas épocas del año, principalmente hacia la zona de la des-

TABLA 1

Variación de las características físicas, químicas, microbiológicas y estructurales del hábitat del estero Nonguén

Variation of the physical, chemical, microbiological and structural features of the Nonguén stream habitat

\begin{tabular}{|c|c|c|c|c|c|}
\hline \multirow[t]{2}{*}{ Parámetro } & \multicolumn{5}{|c|}{ Estación de muestreo } \\
\hline & 1 & 2 & 3 & 4 & 5 \\
\hline Profundidad media $(\mathrm{cm})$ & $30-50$ & $35-100$ & $20-40$ & $30-50$ & $20-40$ \\
\hline Profundidad máxima $(\mathrm{cm})$ & $60-120$ & $60-120$ & $35-100$ & $60-130$ & $60-80$ \\
\hline Ancho cauce (m) & $7-8$ & $3-6$ & $3-6$ & $3-4$ & $3-6$ \\
\hline Temperatura $\left({ }^{\circ} \mathrm{C}\right)$ & $9,0-13,5$ & $11,0-14,2$ & $10,2-15,6$ & $10,7-16,9$ & $12,2-17,5$ \\
\hline $\mathrm{PH}$ & $6,2-6,8$ & $6,2-7,4$ & $6,1-6,8$ & $6,0-6,9$ & $6,3-7,1$ \\
\hline Conductividad $\left(\mu \mathrm{S} \mathrm{cm}^{-1}\right)$ & $59-78$ & $69-96$ & $88-115$ & $78-118$ & $92-123$ \\
\hline Oxígeno disuelto $\left(\mathrm{mg} / \mathrm{L}^{-1}\right)$ & $8,7-9,8$ & $7,6-9,6$ & $7,5-8,6$ & $6,3-9,5$ & $5,5-9,0$ \\
\hline Coliformes fecales (NMP $\left.100 \mathrm{~mL}^{-1}\right)^{*}$ & 49 & 70 & - & $>24.000$ & 35.000 \\
\hline Fósforo total $\left(\mathrm{mg} \mathrm{L}^{-1}\right)^{*}$ & 0,01 & 0,03 & - & 0,03 & 0,18 \\
\hline Nitrógeno total $\left(\mathrm{mg} \mathrm{L}^{-1}\right)^{*}$ & 0,10 & 0,09 & - & 0,22 & 1,76 \\
\hline $\mathrm{DBO}_{5}\left(\mathrm{mg} \mathrm{L}^{-1}\right)^{*}$ & 0,7 & 0,8 & - & 0,3 & 3,9 \\
\hline Tipo de sustrato & Bolón & Bolón- arena & Gravilla- arena & Arena & Arena \\
\hline Vegetación acuática & Ausente & Ausente & Presente & Presente & Presente \\
\hline Entorno al río & $\begin{array}{l}\text { Vegetación } \\
\text { nativa }\end{array}$ & $\begin{array}{c}\text { Cultivos- } \\
\text { plantaciones }\end{array}$ & Viviendas & Viviendas & Viviendas \\
\hline
\end{tabular}

*Mediciones posteriores al muestreo de peces (EULA 2002) 
TABLA 2

Listado de especies encontradas en el estero Nonguén y su distribución latitudinal en Chile

Species composition of Nonguén stream basin and its latitudinal ranges in Chile

\begin{tabular}{|c|c|c|c|c|}
\hline Familia & Nombre científico & Nombre común & Origen & Distribución latitudinal en Chile \\
\hline Geotridae & Geotria australis Gray, 1851 & Lamprea de bolsa & Nativa & Santiago-Tierra del Fuego $\left(33^{\circ} 30^{\prime}-54^{\circ} \mathrm{S}\right)$ \\
\hline Characidae & Cheirodon galusdae Girard, 1854 & Pocha común & Nativa & San Javier-Lautaro $\left(35^{\circ} 30^{\prime}-38^{\circ} 30^{\prime}\right.$ S) \\
\hline \multirow[t]{3}{*}{ Trichomycteridae } & Trichomycterus areolatus Valenciennes, 1848 & Bagre pintado & Nativa & Illapel-Llanquihue $\left(31^{\circ}-41^{\circ} \mathrm{S}\right)$ \\
\hline & Nematogenys inermis (Guichenot, 1848) & Bagre grande & Nativa & Santiago-Osorno $\left(33^{\circ} 30^{\prime}-40^{\circ} 30^{\prime} \mathrm{S}\right)$ \\
\hline & Bullockia maldonadoi (Eigenmann, 1927) & Bagre de Maldonado & Nativa & Concepción-Lautaro (36 $50^{\circ}-38^{\circ} 30^{\prime}$ S) \\
\hline Galaxiidae & Galaxias maculatus (Jenyns, 1842) & Puye & Nativa & Río Huasco-Tierra del Fuego $\left(28^{\circ} 40^{\prime}-54^{\circ} \mathrm{S}\right)$ \\
\hline \multirow[t]{2}{*}{ Salmonidae } & Oncorhynchus mykiss Walbaum, 1892 & Trucha arcoiris & Introducida & Río Loa-Tierra del Fuego $\left(22^{\circ} 30^{\prime}-54^{\circ} \mathrm{S}\right)$ \\
\hline & Salmo trutta Linneo, 1758 & Trucha café & Introducida & Aconcagua-Tierra del Fuego $\left(33^{\circ}-54^{\circ} \mathrm{S}\right)$ \\
\hline Atherinopsidae & Basilichthys australis Eigenmann, 1927 & Pejerrey & Nativa & 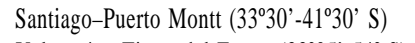 \\
\hline Percicthyidae & Percichthys trucha (Valenciennes, 1833) & Perca trucha, trucha criolla & Nativa & Valparaíso-Tierra del Fuego $\left(33^{\circ} 05^{\prime}-54^{\circ} \mathrm{S}\right)$ \\
\hline Perciliidae & Percilia irwini Eigenmann, 1927 & Carmelita de Concepción & Nativa & Concepción (360 50’ S) \\
\hline Poeciliidae & Gambusia holbrooki (Agassiz, 1859) & Pez mosquito & Introducida & Arica-Tierra del Fuego $\left(19^{\circ}-54^{\circ} \mathrm{S}\right)$ \\
\hline
\end{tabular}

embocadura, donde tendieron a ser mayores en otoño y verano (Fig. 2). En invierno se registró la menor captura a lo largo de todo el eje longitudinal del Nonguén (Fig. 2A) y las máximas se obtuvieron durante el verano (Fig. 2B).

El número de especies de peces aumentó en el sentido de la corriente, variando de 4 en la estación E-1 a 10 en la estación E-5 (Fig. 3), siendo Salmo trutta (Linnaeus, 1758) la única especie que se encuentra a lo largo de todo el estero. Este patrón de distribución de las especies es consistente con la diversidad, la cual mostró un incremento desde el nacimiento del Nonguén hasta su desembocadura (Fig. 4). La diversidad de la estación E-1 fue significativamente menor a la de las estaciones E-2, E-3 y E-5 (prueba de MannWhitney; $Z=-2,309 ; P=0,029)$. Ninguna de las restantes comparaciones entre estaciones de muestreo resultó significativamente distinta. La menor diversidad ocurrió en invierno, excepto en las estaciones E-2 y E-4.

Las especies dominantes variaron espacial y temporalmente, siendo las especies introducidas S. trutta y Oncorhynchus mykiss (Walbaum, 1792) las más frecuentes en la parte alta del estero, principalmente en invierno y verano (Tabla 3 ). En la parte media domina Trichomycterus areolatus, Valenciennes, 1848, prácticamente durante todo el año, mientras que en la desembocadura, existe una alternancia en el dominio dependiendo de la época, de B. maldonadoi en otoño, Basilichthys australis Eigenmann, 1927 en invierno, Galaxias maculatus (Jenyns, 1842) en primavera y Cheirodon galusdae Eigenmann, 1927 y Percichthys trucha (Valenciennes, 1833) en verano. Consistentemente, en E-1 no se detectaron cambios significativos en el aporte porcentual de las diferentes especies entre períodos sucesivos de muestreo. Las diferencias significativas en el rango de abundancias de las distintas especies ocurrieron en las zonas media y baja del río, principalmente entre otoño e invierno, e invierno y primavera (Tabla 4). A pesar de la alternancia de especies dominantes, se mantuvo el patrón de incremento de la riqueza específica desde el nacimiento hasta la desembocadura y desde el invierno hacia el verano.

Los rangos de tamaño corporal del conjunto ictiofaunístico dependen de la interacción entre la época del año y la estación de muestreo (Tabla 5, Fig. 5). Las estaciones E-3, E-4 y E-5 presentan un ensamble con peces de menor talla que los ejemplares capturados en la cabecera del estero (Fig. 5). La asociación íctica de mayor longitud corporal se detectó durante el otoño en la estación E-2 (análisis de varianza ANOVA, $\mathrm{F}_{4,555}=15,85$; $\mathrm{P}<0,001)$, lo cual se asoció a una dominancia de salmónidos (que presentan mayor longitud que las especies nativas). Durante el verano la asociación de peces de menor longitud se encontró en la estación 3, y donde dominaron las especies nativas (análisis de varianza ANOVA, $\mathrm{F}_{3,555}=6,012$; $\mathrm{P}<0,001)$.

El ensamble de cabecera (E-1), dominado por salmonídeos, no varió su rango de tallas durante el año (Tabla 5, Fig. 5). En estaciones donde ocurre una alternancia de especies dominantes entre las introducidas y las nativas (según la época del año), el rango de tallas del conjunto de la ictiofauna varió significativamente (Tabla 5, Fig. 5). Las estaciones más cercanas a la desembocadura (E-4 y E-5), presentaronn los ejemplares más grandes durante el verano, mientras que, en las estaciones de la zona media (E-2 y E-3), los 
ejemplares de mayor tamaño corporal se registraron durante el otoño (Tabla 5, Fig. 5). En ambos casos la variación espacio-temporal se explica por la presencia de salmonídeos de gran talla, particularmente de $S$. trutta.
La biomasa por unidad de esfuerzo del ensamble de peces capturado a lo largo del Nonguén fue menor en invierno y primavera (Fig. 6A), cuando se obtuvo menos de 400 g (372 g en la estación E-

TABLA 3

Composición y biomasa (\%) del ensamble de peces en cada estación de muestreo durante el periodo de estudio

Composition and biomass $(\%)$ of the fish assemblage in each sampling station during the study period

\begin{tabular}{cccccccc}
\hline Estación & Otoño & & Invierno & Primavera & & Verano \\
& Abundancia Biomasa & $\mathrm{n}$ & Abundancia Biomasa & $\mathrm{n}$ & Abundancia Biomasa & $\mathrm{n}$ & Abundancia Biomasa
\end{tabular}
$(\%)$
$(\%)$
$(\%)$
$(\%)$
$(\%)$
(\%)
(\%) $\quad(\%)$

Estación 1

Salmo trutta

Oncorhynchus mykiss

$50,00 \quad 20,44 \quad 21$

$\begin{array}{rrrr}14,29 & 19,79 & 1 & 5,26\end{array}$

5,22

$31,82 \quad 66,31 \quad 7$

Trichomycterus areolatus

$40,48 \quad 53,23 \quad 17$

80,21

$\begin{array}{rrr}5,26 & 5,22 & \\ 73,68 & 68,18 & 14 \\ 15,80 & 2,12 & \\ 5,26 & 24,48 & \end{array}$

$63,64 \quad 20,30 \quad 14$

Nematogenys inermis

$\begin{array}{rrr}2,38 & 23,72 \quad 1\end{array}$

5,26

4,54

$13,39 \quad 1$

Estación 2

Salmo trutta

Oncorhynchus mykiss

Nematogenys inermis

Percichthys trucha

Cheirodon galusdae

Geotria australis

Basilichthys australis

$\begin{array}{rrr}19,20 & 66,35 & 5 \\ 61,50 & 29,99 & 16 \\ 3,80 & 1,05 & 1 \\ 3,80 & 1,90 & 1 \\ 7,70 & 0,06 & 2 \\ 3,80 & 0,65 & 1 \\ - & - & -\end{array}$

$\begin{array}{rrr}11,11 & 43,39 & 1 \\ 22,22 & 43,56 & 2 \\ - & - & - \\ - & - & - \\ 22,22 & 1,49 & 2 \\ - & - & - \\ 44,45 & 11,56 & 4\end{array}$

$\begin{array}{rrr}14,29 & 24,01 & 1 \\ 28,56 & 3,24 & 2 \\ 42,86 & 71,98 & 3 \\ - & - & - \\ 14,29 & 0,77 & 1 \\ - & - & - \\ - & - & -\end{array}$

$\begin{array}{rrr}24,56 & 48,60 & 14 \\ 50,89 & 20,56 & 29 \\ 5,26 & 20,22 & 3 \\ 1,75 & 9,12 & 1 \\ 17,54 & 1,50 & 10 \\ - & - & - \\ - & - & -\end{array}$

Estación 3

Salmo trutta

Oncorhynchus mykiss

$68,87 \quad 5$

$\begin{array}{rrr}- & - & - \\ 7,69 & 56,84 & 1 \\ - & - & -\end{array}$

$\begin{array}{rrr}1,72 & 0,75 & 1 \\ - & - & - \\ 51,73 & 76,67 & 30 \\ 3,45 & 3,40 & 2 \\ 8,62 & 2,53 & 5 \\ 3,45 & 2,26 & 2 \\ 31,03 & 14,39 & 18\end{array}$

$11,11 \quad 60,92 \quad 2$

$10,52 \quad 25,90 \quad 2$

$\begin{array}{rrr}11,11 & - & - \\ 55,56 & 35,52 & 10 \\ - & - & - \\ 5,55 & 0,14 & 1 \\ 27,78 & 3,42 & 5 \\ - & - & -\end{array}$

Estación 4

Salmo trutta

Trichomycterus areolatus

Percichthys trucha

Cheirodon galusdae

Geotria australis

Percilia irwini

Basilichthys australis

Bullokia maldonadoi

Galaxias maculatus

47,37

$\begin{array}{rrr}- & - & - \\ 76,92 & 39,49 & 10\end{array}$

$15,38 \quad 3,67 \quad 2$

31,03

$\begin{array}{rrr}- & - & - \\ 2,15 & 1,50 & 2\end{array}$

$\begin{array}{lll}6,45 & 7,39 & 6\end{array}$

$5,38 \quad 2,56 \quad 5$

$\begin{array}{rrr}- & - & - \\ 15,38 & 6,84 & 2 \\ - & - & - \\ 61,54 & 27,43 & 8 \\ 7,69 & 48,66 & 1 \\ - & - & - \\ 7,69 & 15,47 & 1 \\ - & - & - \\ 7,69 & 1,60 & 1\end{array}$

\begin{tabular}{|c|c|}
\hline 5,26 & 73,73 \\
\hline 10,53 & 5,26 \\
\hline 2,63 & 2,65 \\
\hline 21,05 & 3,21 \\
\hline - & - \\
\hline 13,16 & 3,67 \\
\hline & - \\
\hline 2,63 & 0,86 \\
\hline 44,74 & 10,62 \\
\hline
\end{tabular}

$\begin{array}{rrr}15,00 & 80,52 & 3 \\ 45,00 & 12,13 & 9 \\ - & - & - \\ 10,00 & 0,61 & 2 \\ - & - & - \\ 25,00 & 5,02 & 5 \\ - & - & - \\ - & - & - \\ 5,00 & 1,72 & 1\end{array}$

Estación 5

Salmo trutta

Oncorhynchus mykiss

Trichomycterus areolatus

$\begin{array}{rrr}3,10 & 74,50 & 1 \\ - & - & - \\ 6,30 & 2,00 & 2 \\ - & - & - \\ - & - & - \\ 9,40 & 3,20 & 3 \\ 3,10 & 4,20 & 1 \\ 18,80 & 1,90 & 6 \\ 59,40 & 14,20 & 19\end{array}$

\begin{tabular}{rrrrrrrrr}
- & - & - & 18,75 & 71,40 & 3 & 3,80 & 15,50 & 2 \\
- & - & - & 6,25 & 14,40 & 1 & 19,20 & 72,70 & 10 \\
- & - & - & 18,75 & 4,90 & 3 & 3,80 & 0,10 & 2 \\
- & - & - & 6,25 & 4,30 & 1 & 21,20 & 10,00 & 11 \\
35,71 & 19,18 & 5 & 6,25 & 0,40 & 1 & 32,70 & 0,90 & 17 \\
- & - & - & 12,50 & 1,50 & 2 & 5,80 & 0,10 & 3 \\
42,86 & 77,01 & 6 & - & - & - & 3,80 & 0,60 & 2 \\
7,14 & 0,81 & 1 & - & - & - & 9,60 & 0,10 & 5 \\
- & - & - & - & - & - & - & - & - \\
14,28 & 3,00 & 2 & 31,25 & 3,10 & 5 & - & - & - \\
\hline
\end{tabular}

\begin{tabular}{rrrrrrrrr}
- & - & - & 18,75 & 71,40 & 3 & 3,80 & 15,50 & 2 \\
- & - & - & 6,25 & 14,40 & 1 & 19,20 & 72,70 & 10 \\
- & - & - & 18,75 & 4,90 & 3 & 3,80 & 0,10 & 2 \\
- & - & - & 6,25 & 4,30 & 1 & 21,20 & 10,00 & 11 \\
35,71 & 19,18 & 5 & 6,25 & 0,40 & 1 & 32,70 & 0,90 & 17 \\
- & - & - & 12,50 & 1,50 & 2 & 5,80 & 0,10 & 3 \\
42,86 & 77,01 & 6 & - & - & - & 3,80 & 0,60 & 2 \\
7,14 & 0,81 & 1 & - & - & - & 9,60 & 0,10 & 5 \\
- & - & - & - & - & - & - & - & - \\
14,28 & 3,00 & 2 & 31,25 & 3,10 & 5 & - & - & - \\
\hline
\end{tabular}

Percichthys trucha

Cheirodon galusdae

Percilia irwini

Basilichthys australis

Gambusia holbrooki

Bullokia maldonadoi

Galaxias maculatus 
(A)

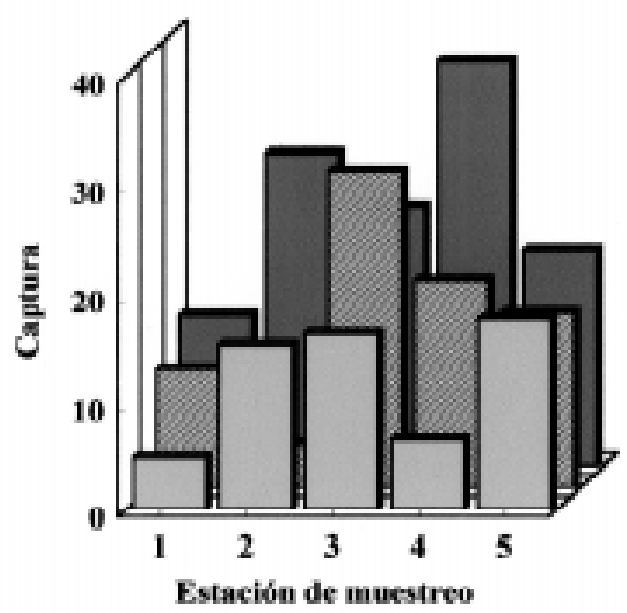

(B)

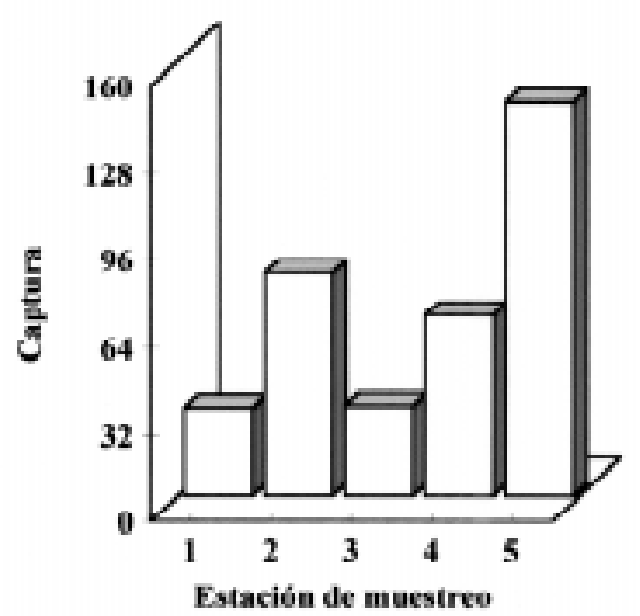

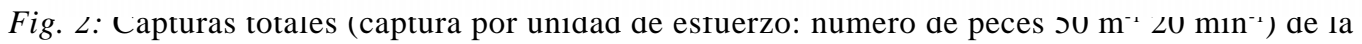
ictiofauna registrada en el estero Nonguén: (A) capturas registradas durante el invierno (color sólido claro), primavera (trama) y otoño (color sólido oscuro); (B) capturas registradas durante el verano.

Total capture (capture per unit of effort: number of fish $50 \mathrm{~m}^{-1} 20 \mathrm{~min}^{-1}$ ) of the fish fauna of Nonguén stream: (A) Winter (solid light), Spring (hatched), and Autumn (dark solid) collections; (B) Summer collections.

2). Los mayores valores de biomasa se obtuvieron durante el verano en la estación E-5 (6187 g) y otoño en la estación E-2 (2.744 g) (Fig. 6B). Este incremento en la biomasa está definido principalmente por los salmónidos y sus oscilaciones se deben a las fluctuaciones en las capturas (Tabla 3). En aquellas estaciones de muestreo en que no aparecen salmónidos, adquiere importancia la biomasa de las especies nativas, excepto en la estación E-2 durante la primavera, que habiéndose capturado un $43 \%$ de salmónidos. Estos fueron individuos muy pequeños, representando tan sólo un $27,2 \%$ de la biomasa. La disminución de la biomasa y abundancia durante primavera (noviembre), parece estar asociada al arrastre de individuos de pequeño tamaño durante las crecidas invernales.

Sólo la baja biomasa de invierno y primavera se relacionó significativamente con variables del ambiente (regresión lineal múltiple, BPUE = $-28,82-0,28 *$ profundidad máxima $-03,94 *$ ancho $+10,59 *$ oxígeno disuelto $; \mathrm{R}^{2}=0,925 ; \mathrm{P}=$ $0,0009)$. Esta relación sugiere indica que los los peces más pequeños, predominantes en esas épocas del año, se distribuyen en sectores del estero más oxigenados, con menor profundidad y ancho.

\section{DISCUSIÓN}

Dado que en todo el país existen aproximadamente 40 especies nativas de agua dulce (Dyer 2000),

\section{TABLA 4}

Cambios en el rango de abundancias de especies entre períodos de muestreo sucesivos a lo largo del estero Nonguén. La letra $\mathrm{Z}$ corresponde al estadígrafo de la prueba de Kolmogorov-Smirnov

Changes in species abundance ranges between successive seasons along Nonguen stream. Letter Z corresponds to the Kolmogorov-Smirnov's statistics

\begin{tabular}{|c|c|c|c|c|c|}
\hline Período & E-1 & E-2 & E-3 & E-4 & E-5 \\
\hline \multicolumn{6}{|c|}{ Otoño-Invierno } \\
\hline $\mathrm{n}$ & 49 & 35 & 32 & 106 & 46 \\
\hline $\mathrm{Z}$ & 0,875 & 1,425 & 2,126 & 2,386 & 1,769 \\
\hline $\mathrm{P}$ & 0,428 & 0,034 & $<0,001$ & $<0,001$ & 0,004 \\
\hline \multicolumn{6}{|c|}{ Invierno-Primavera } \\
\hline $\mathrm{n}$ & 26 & 16 & 71 & 51 & 30 \\
\hline $\mathrm{Z}$ & 0,476 & 1,039 & 1,603 & 1,405 & 1,366 \\
\hline $\mathrm{P}$ & 0,977 & 0,230 & 0,012 & 0,039 & 0,048 \\
\hline \multicolumn{6}{|c|}{ Primavera-Verano } \\
\hline $\mathrm{n}$ & 41 & 64 & 76 & 58 & 68 \\
\hline $\mathrm{Z}$ & 0,848 & 0,814 & 1,150 & 1,600 & 1,093 \\
\hline $\mathrm{P}$ & 0,469 & 0,522 & 0,142 & 0,012 & 0,183 \\
\hline \multicolumn{6}{|c|}{ Verano-Otoño } \\
\hline $\mathrm{n}$ & 64 & 83 & 37 & 113 & 84 \\
\hline $\mathrm{Z}$ & 0,691 & 0,254 & 0,782 & 3,156 & 3,178 \\
\hline $\mathrm{P}$ & 0,726 & 1,000 & 0,573 & $<0,001$ & $<0,001$ \\
\hline
\end{tabular}


la presencia de nueve de ellas $(23 \%)$ en un sistema de pequeña envergadura como el estero Nongúen, es bastante llamativa, más aún si se considera que otras cuencas hidrográficas de gran extensión, tales como la del río Biobío ( 380 km de largo) o del río Itata $(100 \mathrm{~km})$ presentan riquezas de especies levemente superiores (18 y 11 especies, respectivamente; Campos et al. 1993, Habit 1998).

Parte de esta riqueza específica del Nongúen se explica por el alto número de especies que presenta la cuenca del río Andalién, del cual es tributario. El sistema hidrográfico del Andalién
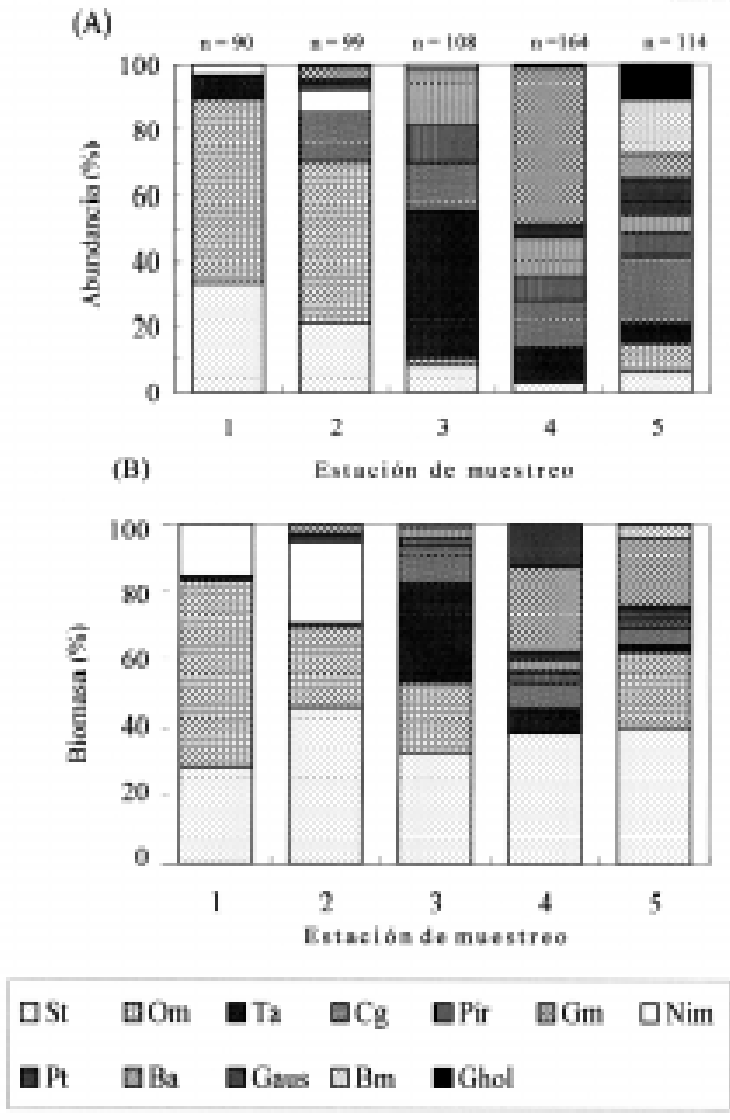

Fig. 3: Contribución de cada especie (porcentaje) en (A) abundancia y (B) biomasa en el ensamble a lo largo del estero Nonguén. G. australis (Gaus), $N$. inermis $(\mathrm{Nim})$, C. galusdae $(\mathrm{Cg})$, S. trutta $(\mathrm{St})$, B. maldonadoi $(\mathrm{Bm}), P$. trucha $(\mathrm{Pt}), P$. irwini (Pir), O. mykiss (Om), G. holbrooki (Ghol), B. australis $(\mathrm{Ba})$, G. maculatus $(\mathrm{Gm})$, T. areolatus (Ta).

Species contribution (percentage) in (A) abundance and (B) biomass to the assemblage along the Nonguén stream. $G$. australis (Gaus), N. inermis (Nim), C. galusdae $(\mathrm{Cg}), S$. trutta $(\mathrm{St})$, B. maldonadoi $(\mathrm{Bm})$, P. trucha $(\mathrm{Pt})$, P. irwini (Pir), O. mykiss (Om), G. holbrooki (Ghol), B. australis (Ba), G. maculatus $(\mathrm{Gm})$, T. areolatus $(\mathrm{Ta})$.

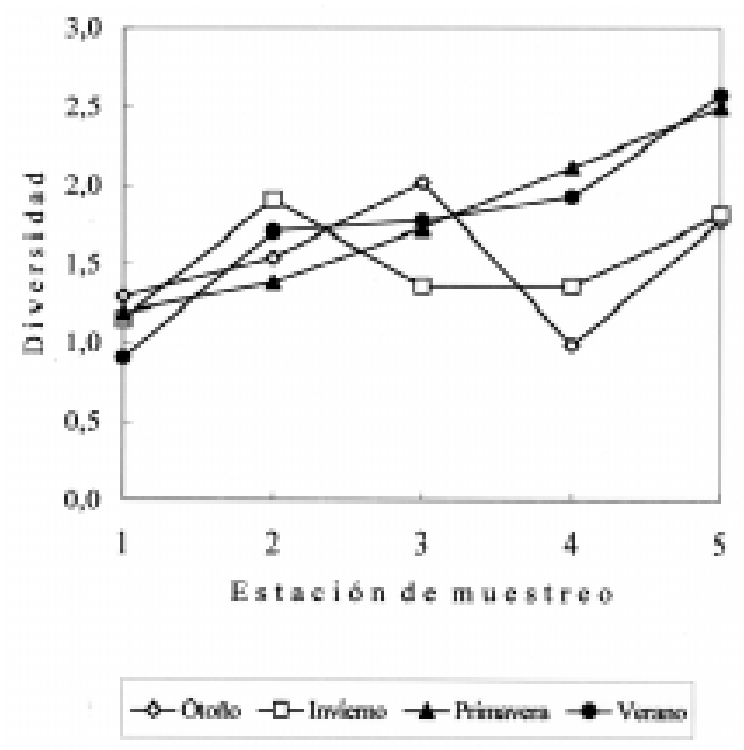

Fig. 4: Diversidad estimada en el estero Nonguén en las campañas de muestreo durante el período de estudio.

Estimated diversity of Nonguén stream per sampling campaign during the study period.

tiene en total 19 especies de peces dulceacuícolas, más ocho estuarinas que aparecen exclusivamente en las áreas cercanas a su desembocadura (Ruiz 1993). Por otra parte, la alta diversidad de peces en el Nonguén queda explicada por la gran variedad de hábitats que este presenta a lo largo de su desarrollo. De hecho, la corta longitud del estero Nonguén, sumado a una pendiente fuerte (Jaque 1995), genera una marcada diferencia entre su zona ritral, media y potamal (confluencia con el río Andalién). Esto implica que el conjunto ictiofaunístico presente un patrón de zonación, con un ensamble característico de la zona de ritrón, dominado por salmónidos, y otro de la zona media y potamal, dominado por especies nativas. Esta zonación se manifiesta, al contrario de los grandes sistemas fluviales (Callow \& Petts 1994, Wolter \& Bischoff 2001), en que los peces de mayor talla se ubican en los tramos altos, en tanto que el ensamble de la zona media e inferior está constituida por ejemplares de mediano a pequeño tamaño corporal (longitud total media de 78,46 mm), característicos de la fauna íctica nativa (Vila et al. 1999a). De esta forma, las zonas de alta calidad ambiental son dominadas por especies introducidas, en tanto, que las especies nativas, incluidas aquellas en peligro de extinción (excepto $N$. inermis), están restringidas al 


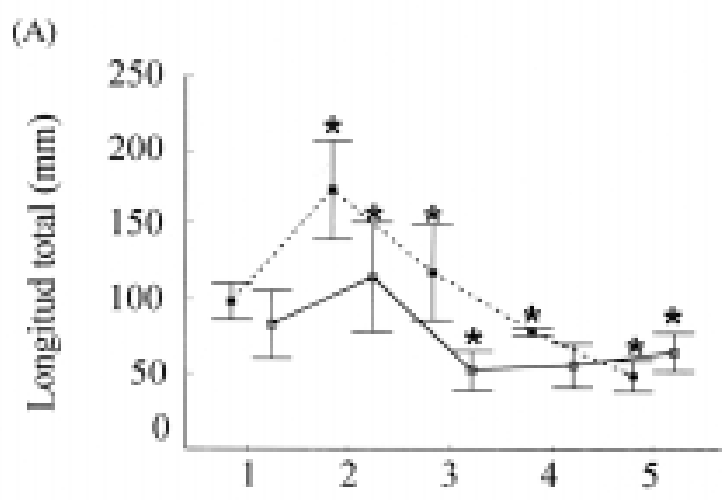

(B)

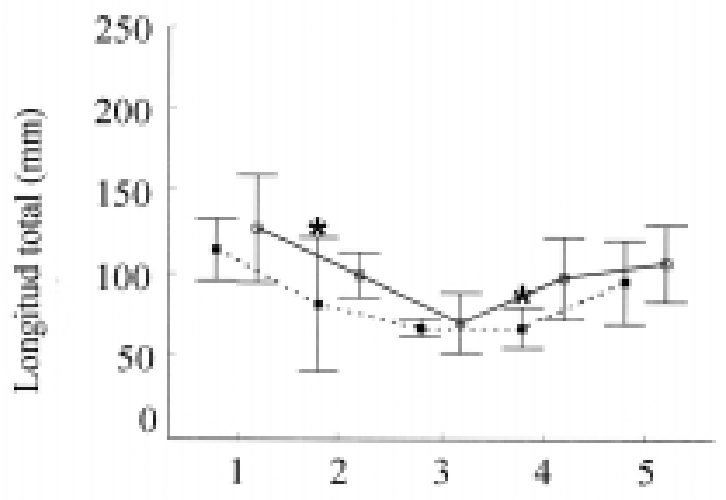

\section{Estación de muestreo}

Fig. 5: Evolución de la longitud total (mm) del ensamble de peces en las estaciones de muestreo del estero Nonguén durante (A) otoño (cuadrado negro) e invierno (cuadrado blanco), y (B) primavera (cuadrado negro) y verano (cuadrado blanco); *las comparaciones de Bonferroni $(0,05)$ corresponden al orden de la campaña posterior para cada estación de muestreo. Las barras representan el error estándar.

Evolution of the Total length ( $\mathrm{mm}$ ) of the fish assemblage in the sampling stations at Estero Nonguén during (A) Autumn (black square) and Winter (white square), (B) Spring (black square) and Summer (white square);

*Bonferroni comparisons (0.05) correspond to the order of the sampling schedule after the each sampling station. Vertical lines show one standard error.

tramo de mayor deterioro del sistema. Es probable que en la situación original (sin especies introducidas), la zona alta del estero se encontrara dominada principalmente por siluriformes nativos, los cuales estarían siendo desplazados por los salmonídeos por competencia y/o depredación. Aún cuando en Chile no existen estudios que hayan cuantificado el efecto de estos peces sobre la ictiofauna local (Dyer 2000), se ha citado su posible efecto negativo a través de la depredación directa, hábitos alimentarios similares, ocupación del espacio y efectos conductuales inhibitorios (Campos 1970, Vila et al. 1999b). A nivel mundial las especies introducidas han sido consideradas como una de las mayores amenazas para la biodiversidad nativa (Aparicio et al. 2000, Walser et al. 2000, Boehn \& Amundsen 2001). En el sistema estudiado, las especies nativas coexisten la mayor parte del año con los juveniles de los dos salmonídeos, por lo que es probable que ocurra competencia por alimento y espacio. En los sitios y épocas en que los nativos coexisten con salmónidos de gran talla, el efecto negativo podría deberse a la depredación. Estas hipótesis podrían ser puestas a prueba experimentalmente en un sistema fluvial de las características del Nonguén, que por su envergadura facilitan los estudios de tipo manipulativo.

Al igual que en otros sistemas fluviales chilenos (Duarte et al. 1971, Campos 1985, Campos et al. 1993, Ruiz 1993, Habit 1998), el Nonguén presenta un aumento en la riqueza específica a lo largo de su curso. Sin embargo, el incremento característico de la abundancia de peces en las partes bajas sólo se manifiesta en verano. Las variaciones en la abundancia y distribución de los peces del Nonguén parecen reflejar los desplazamientos asociados a la ontogenia, reproducción y/o alimentación de los peces (Grenouillet \& Pont 2001, Methven et al. 2001, Taylor \& Warren 2001), más que a las alteraciones del sistema. Los cambios estacionales en las abundancias son más apreciables en especies de gran movilidad o de comportamiento migratorio (Winemiller \& Jepsen 1998), que en este estero corresponden a O. mykiss, $S$. trutta, Geotria autralis Gray, 1851 y $G$. maculatus. Esta última probablemente corresponda a una población diadrómica (sensu Campos et al. 1993) que aparece principalmente representada en su estado juvenil durante primavera, siendo los adultos escasos en el sistema. De igual forma, las especies no migratorias $P$. trucha y B. australis, pueden presentar movimientos locales longitudinales, necesarios para completar sus ciclos de vida. Esto ha sido descrito para peces potamales que viven asociados durante su primer año de vida a hábitats ribereños someros y luego requieren desplazarse a hábitats de mayor profundidad, ubicados en el canal central de ríos de mayor orden (Taylor \& Warren 2001). En cambio, las especies bentónicas como los bagres, con baja capacidad de desplazamiento, son menos variables en el tiempo.

En este sistema fluvial, la mayor variación en la abundancia, biomasa y distribución de la 
ictiofauna se asocia a la época de reproducción, lo cual ha sido reportado también para ensambles de estuarios (Methven et al. 2001) y ríos de mayor orden (Wolter \& Bischoff 2001). En el Nonguén, durante el verano (febrero) y a comienzos de otoño (marzo) se encuentran ejemplares adultos de gran tamaño a lo largo del estero, particularmente individuos reproductores de truchas, los que ingresarían desde el curso principal del río Andalién a este sistema para la reproducción. Ello explica el aumento de la biomasa de peces durante el verano, la cual disminuye durante invierno (julio) y primavera (noviembre), cuando los adultos abandonan este sistema de bajo orden y buscan refugio y alimento en aguas más profundas. La disminución de biomasa íctica y de la longitud media del ensamble detectada en invierno podría responder a un evento de reclutamiento en otoño (e.g., B. australis en E-4, Tabla 2). De esta forma, los principales habitantes del estero durante invierno son juveniles. El bajo número de ejemplares durante la primavera parece estar asociado al arrastre de individuos pequeños o juveniles durante las crecidas de invierno, lo que podría corresponder a un mecanismo de dispersión pasiva, mediante el cual colonizan cursos fluviales de mayor orden (Congdon 1995).

Los resultados sugieren que la alteración antrópica del área potamal no modifica significativamente los patrones característicos de

(A)

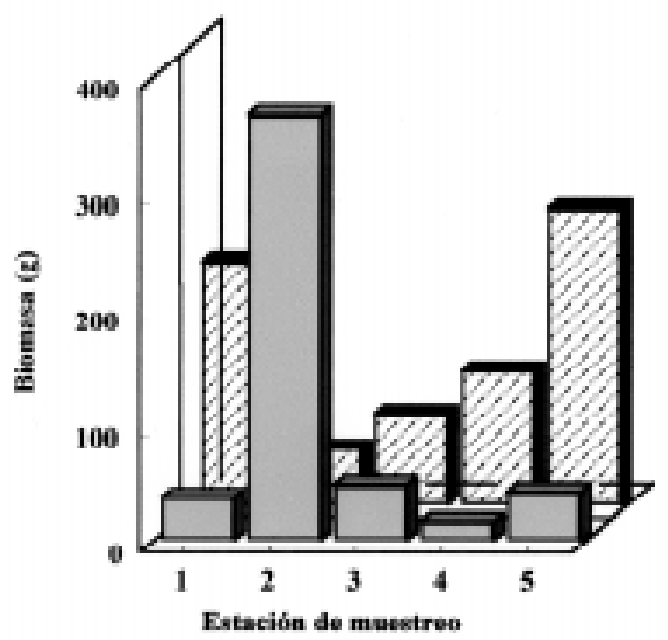

la ictiofauna de sistemas fluviales, y que sus variaciones espacio-temporales se relacionan principalmente con movimientos asociados a la reproducción de las distintas especies. Dada la biología de las especies salmonídeas introducidas y su mayor tamaño corporal respecto de las nativas, éstas determinan la variabilidad espaciotemporal del ensamble de peces. Sin embargo, el estero Nonguén constituye un sistema de reproducción y crianza de juveniles, tanto de especies nativas como introducidas, las cuales conforman asociaciones permanentes en este sistema fluvial. A pesar del grado de intervención del sistema en su zona media e inferior, existe en dicha zona un importante número de especies nativas, por lo cual el estero Nonguén presenta una alto valor para la conservación de la fauna nativa.

\section{AGRADECIMIENTOS}

Agradecemos a Bryan Dyer, Oscar Parra y Claudio Valdovinos por sus valiosas observaciones al manuscrito, así como a los correctores anónimos que aportaron interesantes ideas. Agradecemos también a Waldo San Martín por su ayuda en el trabajo de campo. Este trabajo fue financiado por el Proyecto Diprode 9904072 de la Universidad del Bío-Bío y Proyecto DIUC 200.113.054-1.3 Universidad de Concepción.

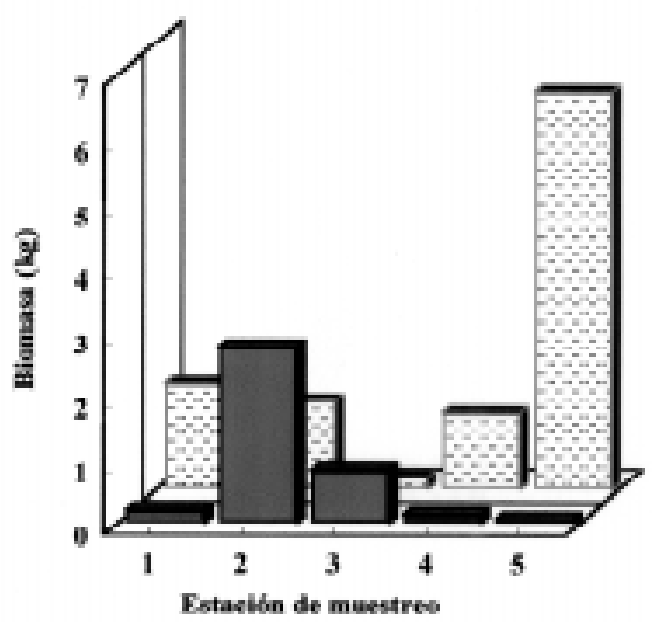

Fig. 6: Biomasa total (biomasa por unidad de esfuerzo: $\mathrm{g}_{0} \mathrm{~m}^{-1} 20 \mathrm{~min}^{-1}$ ) de la ictiofauna registrada en el estero Nonguén. (A) Biomasa (g) registrada durante el invierno (color sólido) y primavera (trama); (B) biomasa (kg) registrada durante otoño (color sólido) y verano (trama).

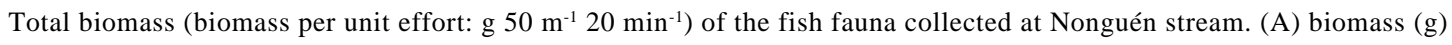
registered during Winter (solid) and Spring (hatched); (B) biomass (kg) registered during Autumn (solid) and Summer (hatched). 
TABLA 5

Resultados del análisis de la varianza (ANOVA) completo y análisis de los efectos simples efectuado sobre la longitud total de los ejemplares del ensamble de peces capturados en las cinco estaciones de muestreo durante el periodo de estudio. La longitud total se transformó mediante el $\log _{10}$. Las comparaciones de Bonferroni $(\alpha=0,05)$ corresponden al orden de las estaciones de muestreo: $\mu_{1}$ es la media de la estación E-1, $\mu_{3}$ es la media de la estación E-3

Results of the complete variance analysis (ANOVA) and the analysis of "simple effects" on the total length of the specimens of the fish assemblage captured in the five sampling stations during the study period. The total length is $\log _{10}$ transformed. The Bonferroni comparisons $(\alpha=0,05)$ are in the same order of the sampling stations: $\mu_{1}$ is the mean of station E-1, $\mu_{3}$ is the mean of station E-3

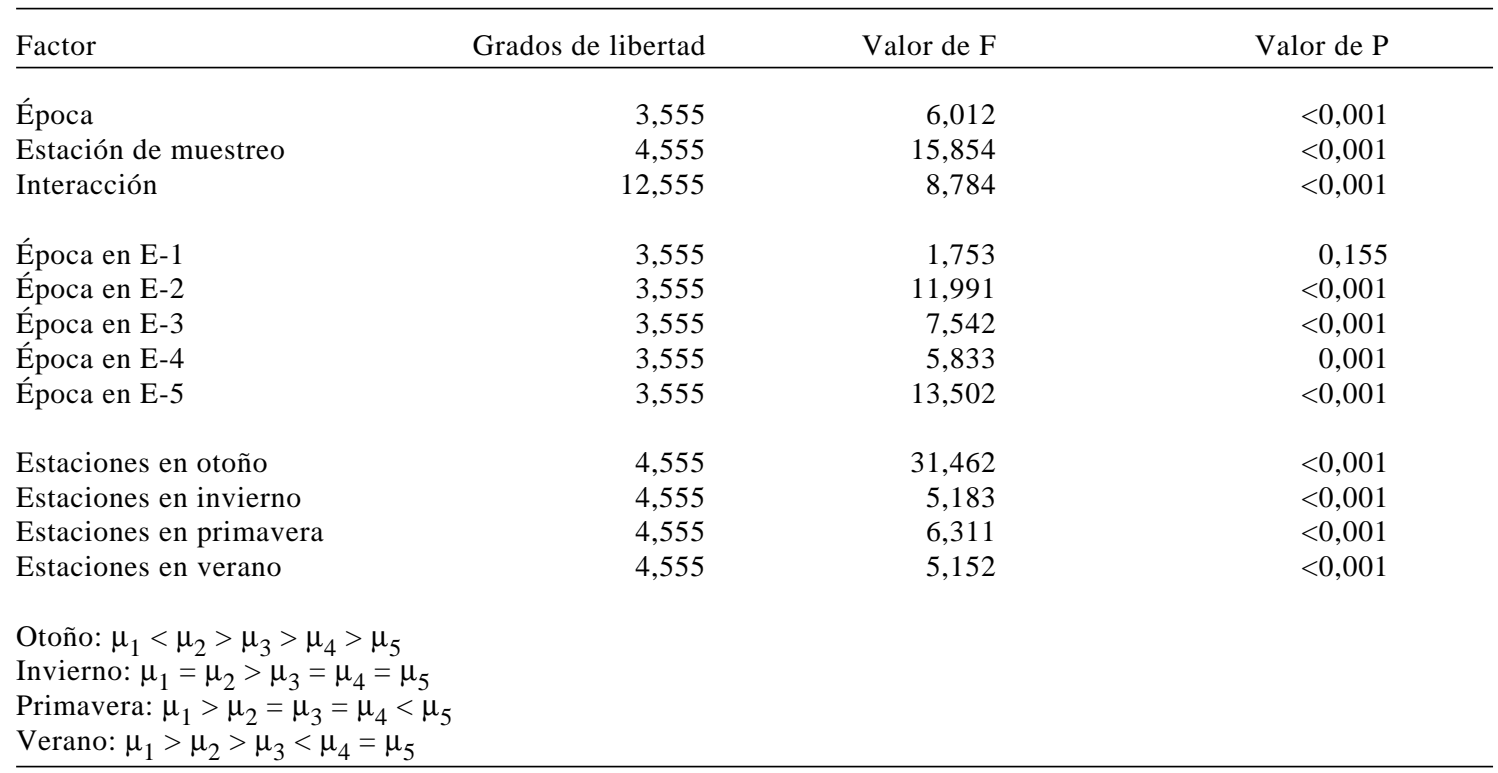

\section{LITERATURA CITADA}

APARICIO E, M VARGAS, J OLMO \& A DE SOSTOA (2000) Decline of native freshwater fishes in a Mediterranean watershed on the Iberian Peninsula: a quantitative assessment. Environmental Biology of Fishes 59: $11-19$.

ARRATIA G (1978) Comentario sobre la introducción de peces exóticos en aguas continentales de Chile. Revista de Ciencias Forestales (Chile) 1: 21 -30.

ARRATIA G (1983) Preferencias de hábitat de peces siluriformes de aguas continentales de Chile (Fam. Diplomystidae y Trichomycteridae). Studies of Neotropical Fauna and Environment 18: 217-237.

BENNINGTON C \& W THAYNE (1994) Use and misuse of mixed model analysis of variance in ecological studies. Ecology 75: 717-722.

BOEHN T \& P AMUNDSEN (2001) The competitive edge of an invading specialist. Ecology 82: 2150-2163.

CAMPOS H (1970) Introducción de especies exóticas y su relación con los peces de agua dulce de Chile. Noticiario Mensual del Museo Nacional de Historia Natural (Chile) 162: 3-9.
CAMPOS H (1985) Distribution of the fishes in the Andean rivers in the south of Chile. Archives of Hydrobiology 104: 169-191.

CAMPOS H, VH RUIZ, JF GAVILÁN \& F ALAY (1993) Peces del río Biobío. Serie Publicaciones de Divulgación EULA, Editorial Universidad de Concepción, Concepción, Chile. 100 pp.

CAMPOS H, G DAZAROLA, B DYER, L FUENTES, JF GAVILÁN, L HUAQUÍN, G MARTÍNEZ, R MELÉNDEZ, G PEQUEÑO, F PONCE, VH RUIZ, W SIEFELD, D SOTO, R VEGA \& I VILA (1998) Categorías de conservación de peces nativos de aguas continentales de Chile. Boletín del Museo Nacional de Historia Natural (Chile) 47: 101-122.

CALLOW P \& G PETTS (eds) (1994) The river handbook: hydrological and ecological principles. Blackwell Science, Oxford, United Kingdom. 523 pp.

CONGDON B (1995) Unidirectional gene flow and maintenance of genetic diversity in mosquitofish Gambusia hobrooki (Teleostei; Poecilidae). Copeia 1995: 162-172.

DUARTE W, R FEITO, C JARA \& AE ORELLANA (1971) Ictiofauna del sistema hidrográfico del río Maipo. Boletin del Museo Nacional de Historia Natural (Chile) 32: 227-268. 
DYER B (2000) Systematic review and biogeography of the freshwater fishes of Chile. Estudios Oceanológicos (Chile) 19: 77-98.

EULA (2002) Diagnóstico y zonificación del Parque Metropolitano Fundo Nonguén. Informe Línea de Base, Oficina Asistencia Técnica, Centro de Ciencias Ambientales EULA-Chile, Universidad de Concepción, Concepción, Chile. 258 pp.

GARCÍA-JALÓN D, M MAYO, F HERVELLA, E BARCELO \& T FERNÁNDEZ (1993) Principios y técnicas de gestión de la pesca en aguas continentales. Ediciones Mundi-Prensa, Madrid, España. 247 pp.

GRENOUILLET G \& D PONT (2001) Juvenile fishes in macrophyte beds: influence of food resources, habitat structure and body size. Journal of Fish Biology 59: 939-959.

HABIT E (1998) Peces. En: Parra O \& E Habit (eds) Estudio de la Línea de Base para la Evaluación de Impacto Ambiental del Complejo Industrial Forestal Itata. Universidad de Concepción, Editorial Aníbal Pinto, Concepción, Chile. 172 pp.

HABIT E \& O PARRA (2001) Impactos ambientales de los canales de riego sobre la fauna de peces. Ambiente y Desarrollo (Chile) 17: 50-56.

ILLIES J \& L BOTOSANEANU (1963) Problemes et methodes de la classification de la zonation ecologique des eaux courantes, considerees surtout du point de vue faunistique. Mitteilungen Internationalen Vereinigung für Theoretische und Angewandte Limnologie 12: 1-57.

JAQUE E (1994) Problemas ambientales en un área de expansión urbana. Cuenca del estero Nonguén. Concepción, Chile. Revista Geográfica de Chile Terra Australis 39: 65-78.

JAQUE E (1995) Análisis integrado de los sistemas naturales de la cuenca del río Andalién: bases para la planificación ecológica del territorio de la cuenca. Tesis Doctoral, Centro EULA, Universidad de Concepción, Concepción, Chile. ix + 267 pp.
METHVEN DA, RL HAEDRICH \& GA ROSE (2001) The fish assemblage of a Newfoundland estuary: diel, monthly and annual variation. Journal of Fish Biology 52: 669-687.

RUIZ VH (1993) Ictiofauna del río Andalién (Concepción, Chile). Gayana Zoología (Chile) 57: 109-278.

SPSS INC (1988) SPSS-X user's guide. SPSS Inc., Chicago, Illinois. $1.072 \mathrm{pp}$.

TAYLOR CM \& ML WARREN (2001) Dynamics in species composition of stream fish assemblages: environmental variability and nested subsets. Ecology 82: $2320-2330$.

VANNOTE RL, G MINSHALL, K CUMMINS, JR SEDELL \& CE CUSHING (1980) The river continuum concept. Canadian Journal of Fisheries and Aquatic Sciences 37: 130-137.

VILA I, L FUENTES \& M CONTRERAS (1999a) Peces límnicos de Chile. Boletín del Museo Nacional de Historia Natural (Chile) 48: 61-75.

VILA I, L FUENTES \& M CONTRERAS (1999b) Ictiofauna en los sistemas límnicos de la Isla Grande, Tierra del Fuego, Chile. Revista Chilena de Historia Natural 72: 273-284.

WALSER C, B FALTERMAN \& H BART (2000) Impact of introduced rough shiner (Notropis baileyi) on the native fish community in the Chattahoochee river system. American Midland Naturalist 144: 393-405.

WELCOMME RL (1985) River fisheries. FAO Fisheries Technical Paper 262: 1-318.

WINEMILLER KO \& DB JEPSEN (1998) Effects of seasonality and fish movement on tropical river food webs. Journal of Fish Biology 53: 267-296.

WOLTER C \& A BISCHOFF (2001) Seasonal changes of fish diversity in the main channel of large lowland River Oder. Regulated Rivers: Research \& Management 17: 595-608.

WOOTTON RJ (1990) Ecology of teleost fishes. Chapman and Hall, New York, New York. 404 pp.

ZAR J (1984) Bioestatistical analysis. Second edition. Prentice-Hall, Englewood Cliffs, New Jersey. 718 pp.

Editor Asociado: Patricio Ojeda

Recibido el 7 de junio de 2002; aceptado el 22 de octubre de 2002 